\title{
Potential of Radiation-Induced Cellular Stress for Reactivation of Latent HIV-1 and Killing of Infected Cells
}

\author{
Sergey lordanskiy ${ }^{1,2}$ and Fatah Kashanchi ${ }^{2}$
}

\begin{abstract}
The use of highly active antiretroviral therapy against HIV-1 for last two decades has reduced mortality of patients through extension of nonsymptomatic phase of infection. However, HIV-1 can be preserved in longlived resting $\mathrm{CD}^{+} \mathrm{T}$ cells, which form a viral reservoir in infected individuals, and potentially in macrophages and astrocytes. Reactivation of viral replication is critical since the host immune response in combination with antiretroviral therapy may eradicate the virus (shock and kill strategy). In this opinion piece, we consider potential application of therapeutic doses of irradiation, the well-known and effective stress signal that induces DNA damage and activates cellular stress response, to resolve two problems: activate HIV-1 replication and virion production in persistent reservoirs under cART and deplete infected cells through selective cell killing using DNA damage responses.
\end{abstract}

$\mathbf{H}$ IV-1 CAN BE preserved in long-lived resting $\mathrm{CD}^{+} \mathrm{T}$ cells, which form a viral reservoir in infected individuals. This reservoir may persist for many years even though the patients are usually treated with highly active antiretroviral therapy (HAART), and viral population can be recovered once HAART is stopped. Thus, the selective activation of latently HIV-infected cells resulting in the replication of proviral genome is critical to make infected cells recognized by the immune system. The host immune response may eliminate the virus following reactivation (shock and kill strategy). Another important issue is selective activation of apoptosis in the infected cells after reactivation. This might increase the chance of infected cells to be killed without death of uninfected T cells. Additionally, elicitation of the treatment that can directly eliminate latently HIVinfected cells within the pool of resting $\mathrm{T}$ cells is also important for effective depletion of viral reservoirs.

The major problem in eradication of HIV from the patients is the lack of therapeutic approaches to recognize the latent HIV-1 provirus and to eliminate latently infected cells. Relatively low frequency of latently infected $\mathrm{T}$ cells in patients $\left(\sim 1 / 10^{5-} 10^{6}\right.$ in the resting $\mathrm{CD} 4^{+} \mathrm{T}$-cell population $\left.{ }^{1}\right)$ and lack of biomarkers for the latent infection make these cells undetectable for the available therapies. Current ideas to use HDAC inhibitors (disulfiram, ${ }^{2}$ vorinostat ${ }^{3}$ ), PKC agonists, or bromodomain inhibitors for virus reactivation are promising since these latency reactivation agents (LRAs) provide robust increase of viral replication without release of proinflammatory cytokines. ${ }^{4,5}$ However, according to recent in vivo studies, treatment with vorinostat, despite transient activation of HIV-1 transcription, does not induce killing of latently infected cells and thus does not reduce the latent reservoir. ${ }^{6,7}$ Therefore, for successful eradication of HIV-1 through selective killing of infected cells within the scope of shock and kill strategy, additional approaches are required.

Ionizing irradiation (IR) is the well-known and effective stress signal that induces DNA damage and activates cellular stress response. The typical X-ray- and $\gamma$-IR-induced DNA lesions cause double-strand breaks that can result in induction of up to four independent repair pathways: homologous recombination, nonhomologous end joining, (NHEJ), alternative NHEJ, and single-strand annealing $\left(\right.$ reviewed in ${ }^{8}$ ). X-ray IR stress usually induces NHEJ machinery, ${ }^{9}$ which is associated with enhanced activity of many cellular factors involved in transcription activation, such as NF- $\kappa \mathrm{B},{ }^{10} \mathrm{Sp} 1,{ }^{11}$ HAT $1,{ }^{12}$ and CBP/p300 whose activation has been shown to lead to SWI/SNF-mediated chromatin remodeling. ${ }^{13,14}$

Earlier studies of the effect of DNA-damaging agents, such as ultraviolet IR, and radiotherapy (X-ray, $\gamma$ IR) on HIV-1 demonstrated that induced SOS-like stress responses enhanced viral gene expression to levels that are similar to those obtained by the tat gene product ${ }^{15-18}$ and induced cell death

${ }^{1}$ National Center for Biodefense and Infectious Diseases, George Mason University, Manassas, Virginia.

${ }^{2}$ Laboratory of Molecular Virology, School of Systems Biology, George Mason University, Manassas, Virginia. 
through chromatin DNA damage. ${ }^{19}$ Both X-ray and $\gamma$-IR have been shown to activate LTR-driven transcription through the activation of NF- $\kappa$ B DNA binding. ${ }^{16,17}$ Our earlier studies indicated that single dose of $\gamma$-IR differently activated infected $\mathrm{T}$ cells compared with the parental uninfected cell lines. Exposition of chronically HIV-1-infected T-cell lines, $\mathrm{ACH} 2$ and $8 \mathrm{E} 5$, to $\gamma$-IR resulted in inappropriate entry of these cells into the $S$ phase and eventual apoptosis, whereas the uninfected parental cells, CEM and A3.01, demonstrated typical response for the transformed cells, namely arrest at G1/S and G2/M phase of cell cycle before repair. ${ }^{20}$ The irradiated chronically infected $\mathrm{T}$ cells also displayed an increased level of HIV-1 mRNA accumulation (both unspliced and spliced) and the viral proteins encoded by the mRNAs, specifically Tat, and Rev, Vpr, and Nef. These data suggested that the nuclear accumulation of HIV-1 proteins, such as Tat and $\mathrm{Vpr}$, may contribute to aberrations in cell cycle regulation, resulting in the loss of $\mathrm{G}_{1} / \mathrm{S}$ checkpoint.

Later, we demonstrated that despite IR having equally induced p53-mediated DNA repair pathways in HIV-1infected and uninfected cells (phosphorylation of Ser9 and Ser15 residues ${ }^{21-23}$ ), the phosphorylation of Ser46, which is responsible for the ability of p53 to induce apoptosis, ${ }^{24}$ was approximately higher in the infected $\mathrm{T}$ cells. These data may potentially explain the lower viability of primary HIV-1infected $\mathrm{CD}^{+} \mathrm{T}$ cells after IR compared with uninfected cells. ${ }^{25,26}$ Other studies reported that the X-ray-treated human colonic carcinoma cells could activate NF-KB-mediated HIV1 transcription in nonirradiated cells through the secretion of cell-activating cytokines, ${ }^{27}$ indicating an indirect effect of IR on HIV-1-infected cells.

Despite dramatic activation of HIV-1 transcription after the treatment with certain combinations of LRAs, ${ }^{5}$ this treatment alone does not provide effective death of infected cells and depletion of latent reservoir. ${ }^{6,7} \mathrm{We}$ recently proposed that IR alone was sufficient to activate the HIV-1 LTR and effectively kill the infected T cells. This killing was especially more pronounced in primary $\mathrm{T}$ cells, including latently infected $\mathrm{CD}^{+}$memory $\mathrm{T}$ cells. We found that cytopathogenic effect of IR on reactivated HIV-1-infected primary $\mathrm{CD} 4^{+} \mathrm{T}$ cells was more than twofold higher than on the uninfected cells. ${ }^{28}$ Recent clinical studies that included analysis of $\mathrm{CD}^{+} \mathrm{T}$-cell count in the blood from cancer patients infected or not with HIV-1 indicated that radiation therapy reduced the $\mathrm{CD} 4^{+}$cell count more dramatically in HIV-1-infected individuals. ${ }^{25,29}$ Treatment with NNRTIbased ART exacerbated this effect. ${ }^{26}$

In addition, another study from Hanauske-Abel and colleagues similarly showed that apoptosis can be preferentially activated in HIV-infected T cells and in peripheral blood mononuclear cells using the topical antifungal ciclopirox and the iron chelator deferiprone, which trigger preferential elimination of infected, but not uninfected, cells. ${ }^{30}$ No viral reemergence was observed even 12 weeks after drug cessation, suggesting elimination of the proviral reservoir. In our experiments with monocytes/macrophages, IR alone did activate HIV-1 LTR, but did not kill infected cells, which is consistent with previously published data about relative resistance of macrophages to cancer radiotherapy ${ }^{31,32}$ Nevertheless, the combination of moderate doses of IR with chemical compound, PKC agonist bryostatin 1, enhanced transcription activation and cell killing in monocyte/macro- phages. ${ }^{28}$ This suggests that the IR effect is cell-type specific and that infected T cells are more prone to apoptosis with IR, whereas IR with LRAs is needed to kill infected myeloid cells.

Another reason to consider low doses of IR as a potential therapeutic approach against latent HIV-1 infection is an impact of stress-induced apoptosis on HIV-1 replication. A recent publication from Zeichner laboratory indicated that cancer chemotherapeutic agents that induce apoptosis dramatically activated HIV-1 replication in latently infected cells. ${ }^{33}$ The authors based their study on the observation that cytotoxic chemotherapy and bone marrow transplantation applied to HIV patients who developed lymphoma and appeared to be relatively more effective at depletion of the HIV1 latent reservoir than LRAs. ${ }^{34,35}$ In fact, treatment of both persistently HIV-1-infected T cells and monocytes with cytotoxic drugs, doxorubicin, etoposide, fludarabine phosphate, or vincristine, was associated with caspase- 3 and -8 activation and therefore apoptosis response that followed dramatic increase of HIV-1 RNA and p24 count.

The only known case of HIV-1 cure in the patient known as Berlin Patient supports the essential role of programmed death of HIV-infected cells for depletion of viral reservoir and, at the same time, applicability of IR as a potential antiHIV treatment. The IR doses used for total body IR in the therapy of lymphoma (two single doses, 2 and 4 Gy) were applied to this patient before transplantation of the bone marrow with CCR5 32 mutation and did not have documented negative effect on other tissues. ${ }^{34,36}$ Meanwhile, the virus was likely eradicated from latent reservoirs, including tissues of the central nervous system, since further prolonged tests did not reveal HIV-1 in both blood and spinal fluid specimens, ${ }^{36}$ whereas two other HIV-1-infected lymphoma patients, called Boston patients, received only chemotherapy before transplantation of wild-type CCR5(+) stem cells. ${ }^{35}$ Both patients displayed relapse of HIV-1 infection in 6 months after stop of ART.

Another potential application of radiotherapy to treat HIV1 infection is the use of radiolabeled antibodies targeting viral envelope glycoproteins exposed on the surface of infected cells.

Antibodies to HIV-1 gp120 and gp41 labeled with alphaemitting radionuclides, 213-bismuth $\left({ }^{213} \mathrm{Bi}\right)$ and rhenium 188 $\left({ }^{188} \mathrm{Re}\right)$, were tested on chronically HIV-1-infected human T cells, ACH-2, and infected peripheral blood mononuclears (PBMCs) in vitro. Selective killing of infected cells after treatment with both antibodies, including dose-dependent $99 \%$ death after ${ }^{213} \mathrm{Bi}$-anti-gp120, was detected. ${ }^{37}$ In vivo experiments with immunodeficient (SCID) mice intraperitoneally injected with human PBMCs from the spleens and peritoneum and infected with HIV-1 also demonstrated 5- to 10-fold dose-dependent reduction of HIV-1 infection after treatment with ${ }^{213} \mathrm{Bi}$-anti-gp41. ${ }^{38}$ Authors declared that this treatment did not cause acute hematologic toxicity in the treated mice. It should be noted here that in spite of demonstrated efficiency of this approach to eliminate productively HIV-1-infected cells, the tested antibodies will not deplete latent reservoir. However, reactivation of latent reservoir before this treatment might potentially increase the level of subsequent depletion of the pool of infected cells.

Our overall view of the HIV-1 transcription and apoptosis of infected cells by IR is depicted in Figure 1. Previous multiple manuscripts have shown that Tat regulates p53 


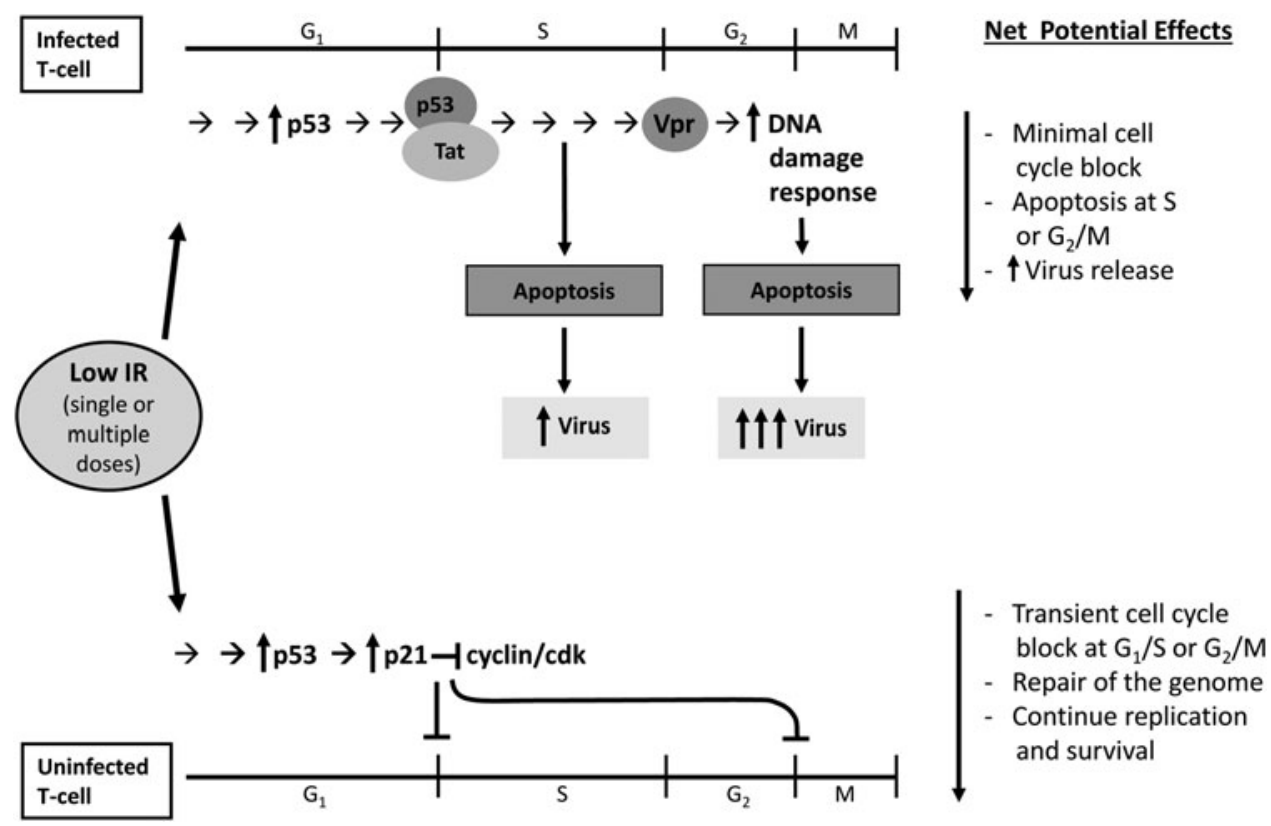

FIG. 1. Effect of irradiation (IR) on infected versus uninfected cells. The diagram is a depiction of what may be occurring in HIV-1-infected T cells versus uninfected cells. Infected cells exposed to low-level IR (either single or multiple low doses) would deregulate the p53 pathway (i.e.p53/Tat binding ${ }^{39,40,49,50}$ ) and allow cells inappropriately to enter the $S$ phase. The cells would then either enter apoptosis at the S or move to the G2 phase, followed by enhanced apoptosis through Vpr. Vpr has been shown to regulate the DNA damage response. ${ }^{45-47}$ The net effect is apoptosis of infected cells with increased viral release. Other potential deregulation of DNA damage response has been observed in HIV-infected cells through the DNA-PK/p53 pathway $^{51-57}$ and other retroviruses, including HTLV. ${ }^{58-62}$ On the other hand, uninfected T cells, when exposed to IR, would activate their normal $\mathrm{p} 53$, leading to activation of $\mathrm{p} 21 / \mathrm{wafl}$ and binding to cyclin/cdk complexes (i.e., cdk2/E, cdk2/A, or cdc2/ B), resulting in blocks at the G1/S or G2/M for DNA repair. After a certain period of time required for DNA repair, cells would resume into the next phase of replication. The model assumes that viral gene expression is increased with IR over time and cellular apoptosis is enhanced by irreversible DNA damage to the infected cells, while the virus is replicating.

function and that it can sequester p53's effect on p21/wafl activation. ${ }^{39,40}$ This would require certain levels of HIV-1 basal transcription and protein synthesis related to viral open reading frames (i.e., inactive methylated Tat; ${ }^{41-43}$ ) potentially present in some latent cells. Whether latent $\mathrm{T}$ cells in vivo have actual full-length transcripts, followed by translation of doubly spliced messages such as Tat in the presence of antiretrovirals, has never been formally shown; however, it is equally difficult to obtain pure latent infected cells for quantitative RT-PCR and almost impossible with current technologies to detect low-level proteins such as Tat in these samples. Therefore, the theoretical assumption is that p53 is deregulated by viral proteins such as Tat, which allows cells to progress inappropriately into the $\mathrm{S}$ phase with IR activation.

Accumulation of cells at the S phase and lack of appreciable DNA repair would cause apoptosis from the $S$ phase. This also follows an assumption that latently infected cells may not be at the G0 phase of cell cycle as no publications to date describe the difference between G0 or early G1 phase in latently HIV-1-infected cells under cART. We presume that cells may be blocked at the early, mid, or late G1 phase with the help of viral noncoding RNAs, such as free TAR RNA. ${ }^{44}$ We recently have shown that TAR is present in cART-treated cells where it activates the TLR3 pathway and deregulates protein kinase $\mathrm{R}$ innate immune response, which could presumably keep cells away from G0 and be maintained at early G1 phase. Alternatively, if cells do pass into the G2 phase, there is high translation of viral proteins such as Vpr, which not only blocks cells at G2/M but also further activates DNA damage response. ${ }^{45-47}$ This would push cells into the G2 phase to have increased viral protein synthesis and ultimately high viral expression, translation, packaging, and apoptosis of the host cell.

Two other related issues, which can equally contribute to apoptosis or lack thereof, include (1) latent cells may already have increased proteins related to DNA damage machinery as a result of viral integration or leaky LTR read through that is not translated (i.e., short TAR RNA), which could contribute to altered DNA damage response and sensitivity to $\mathrm{IR}^{48} ;(2)$ myeloid cells that are inherently differentiated are locked at the early G1 phase (2N) and the IR can increase gene expression, but would have minimal to no effect toward apoptosis of the infected cells at the $\mathrm{S}$ or G2/M, making the therapeutic value of IR alone for apoptosis in these cells not significant. Under these conditions, there may need to be a second agent that pushes infected myeloid cells (inherently more resistant to apoptosis compared with uninfected cells) into the apoptotic pathway (i.e., p53-independent pathway).

On the other hand, uninfected cells can tolerate low levels of IR by activating its wild-type p53 and p21/wafl induction, which would bind to all G1/S cyclin/cdk complexes and inhibit their Rb phosphorylation. This would block cells transiently at the G1/S or the G2/M and allow enough time to repair the damaged DNA, followed by release of cells to move forward into the next stage of replication or simply stay 
quiescent after few passages. Again, whether all forms of T cells are equally sensitive to low level of IR remains to be determined as no such data exist to date (i.e., differences between central memory [TCM] vs. transitional memory [TTM] T cells). In conclusion, the potential for IR activation of the HIV promoter with subsequent apoptosis of infected cells offers an attractive mode of shock and kill; however, for the approach to be fully realized in either AIDS patients under cART or AIDS patients with cancer, it requires not only infectious disease expertise but also a basic understanding of radiation oncology. Along these lines, lessons learned from cancer radiation oncology, which has proven to be effective for more than five decades when using low-level IR treatment, make the approach a manageable solution in immunocompromised patients.

\section{Acknowledgments}

The authors would like to thank the members of the Kashanchi Laboratory, especially Angela Schwab, for assistance with the manuscript and Figure 1. This work was supported by NIH grants, AI043894, AI114490, and AI113140, to F.K. The content is solely the responsibility of the authors and does not necessarily represent the official views of the National Institutes of Health.

\section{Author Disclosure Statement}

No competing financial interests exist.

\section{References}

1. Ho YC, Shan L, Hosmane NN, et al:: Replication-competent noninduced proviruses in the latent reservoir increase barrier to HIV-1 cure. Cell 2013;155:540-551.

2. Xing S, Bullen CK, Shroff NS, et al.: Disulfiram reactivates latent HIV-1 in a Bcl-2-transduced primary CD4+ T cell model without inducing global $\mathrm{T}$ cell activation. $\mathrm{J}$ Virol 2011;85:6060-6064.

3. Archin NM, Espeseth A, Parker D, Cheema M, Hazuda D, Margolis DM: Expression of latent HIV induced by the potent HDAC inhibitor suberoylanilide hydroxamic acid. AIDS Res Hum Retroviruses 2009;25:207-212.

4. Archin NM, Liberty AL, Kashuba AD, et al.: Administration of vorinostat disrupts HIV-1 latency in patients on antiretroviral therapy. Nature 2012;487:482-485.

5. Laird GM, Bullen CK, Rosenbloom DI, et al.: Ex vivo analysis identifies effective HIV-1 latency-reversing drug combinations. J Clin Invest 2015;125:1901-1912.

6. Blazkova J, Chun TW, Belay BW, et al.: Effect of histone deacetylase inhibitors on HIV production in latently infected, resting CD4(+) T cells from infected individuals receiving effective antiretroviral therapy. J Infect Dis 2012; 206:765-769.

7. Ke R, Lewin SR, Elliott JH, Perelson AS: Modeling the effects of vorinostat in vivo reveals both transient and delayed HIV transcriptional activation and minimal killing of latently infected cells. PLoS Pathog 2015;11: e1005237.

8. Curtin NJ: DNA repair dysregulation from cancer driver to therapeutic target. Nat Rev Cancer 2012;12:801-817.

9. Hartlerode AJ, Scully R: Mechanisms of double-strand break repair in somatic mammalian cells. Biochem J 2009; 423:157-168.
10. Aggarwal BB: Nuclear factor-kappaB: The enemy within. Cancer Cell 2004;6:203-208.

11. Iwahori S, Yasui $\mathrm{Y}$, Kudoh $\mathrm{A}$, et al: Identification of phosphorylation sites on transcription factor $\mathrm{Sp} 1$ in response to DNA damage and its accumulation at damaged sites. Cell Signal 2008;20:1795-1803.

12. Lebel EA, Boukamp P, Tafrov ST: Irradiation with heavyion particles changes the cellular distribution of human histone acetyltransferase HAT1. Mol Cell Biochem 2010; 339:271-284.

13. Agbottah E, Deng L, Dannenberg LO, Pumfery A, Kashanchi F: Effect of SWI/SNF chromatin remodeling complex on HIV-1 Tat activated transcription. Retrovirology 2006;3:48.

14. Ogiwara H, Ui A, Otsuka A, et al:: Histone acetylation by CBP and p300 at double-strand break sites facilitates SWI/SNF chromatin remodeling and the recruitment of non-homologous end joining factors. Oncogene 2011;30:2135-2146.

15. Valerie K, Delers A, Bruck C, et al.: Activation of human immunodeficiency virus type 1 by DNA damage in human cells. Nature 1988;333:78-81.

16. Faure E, Cavard C, Zider A, Guillet JP, Resbeut M, Champion S: $\mathrm{X}$ irradiation-induced transcription from the HIV type 1 long terminal repeat. AIDS Res Hum Retroviruses 1995;11:41-43.

17. Smith RA, Ingels J, Lochemes JJ, Dutkowsky JP, Pifer LL: Gamma irradiation of HIV-1. J Orthop Res 2001;19:815-819.

18. Sadaie MR, Tschachler E, Valerie K, et al: : Activation of tat-defective human immunodeficiency virus by ultraviolet light. New Biol 1990;2:479-486.

19. Ogawa Y, Kobayashi T, Nishioka A, et al:: Radiationinduced oxidative DNA damage, 8-oxoguanine, in human peripheral T cells. Int J Mol Med 2003;11:27-32.

20. Clark E, Santiago F, Deng L, et al:: Loss of $\mathrm{G}(1) / \mathrm{S}$ checkpoint in human immunodeficiency virus type 1infected cells is associated with a lack of cyclin-dependent kinase inhibitor p21/Waf1. J Virol 2000;74:5040-5052.

21. Shahbazi J, Lock R, Liu T: Tumor protein 53-induced nuclear protein 1 enhances p53 function and represses tumorigenesis. Front Genet 2013;4:80.

22. Haas RL: Low dose radiotherapy in indolent lymphomas, enough is enough. Hematol Oncol 2009;27:71-81.

23. Yamaguchi T, Matsuda K, Sagiya Y, et al.: p53R2dependent pathway for DNA synthesis in a p53-regulated cell cycle checkpoint. Cancer Res 2001;61:8256-8262.

24. Oda K, Arakawa H, Tanaka T, et al.: p53AIP1, a potential mediator of p53-dependent apoptosis, and its regulation by Ser-46-phosphorylated p53. Cell 2000;102:849-862.

25. Sankatsing SU, Hillebregt MM, Gras L, et al.: Prolonged decrease of CD4+ T lymphocytes in HIV-1-infected patients after radiotherapy for a solid tumor. J Acquir Immune Defic Syndr 2013;62:546-549.

26. Ulrike K, Markus H, Thomas H, et al:: NNRTI-based antiretroviral therapy may increase risk of radiation induced side effects in HIV-1-infected patients. Radiother Oncol 2015; 116:323-330.

27. Faure E: X-rays-induced secretion of cellular factor(s) that enhance(s) HIV-1 promoter transcription in various nonirradiated transfected cell lines. Cell Mol Biol (Noisy-legrand) 1998;44:1275-1292.

28. Iordanskiy S, Van Duyne R, Sampey GC, et al.: Therapeutic doses of irradiation activate viral transcription and induce apoptosis in HIV-1 infected cells. Virology 2015;485:1-15.

29. Siraprapasiri $\mathrm{P}$, Tharavichitkul E, Suntornpong $\mathrm{N}$, et al.: Effect of radiation therapy to immunological and virological 
status in HIV/AIDS-cancer patients, preliminary report. J Med Assoc Thai 2011;94 Suppl 2:S88-S93.

30. Hanauske-Abel HM, Saxena D, Palumbo PE, et al.: Druginduced reactivation of apoptosis abrogates HIV-1 infection. PLoS One 2013;8:e74414.

31. Schmidtke JR, Dixon FJ: The functional capacity of $x-$ irradiated macrophages. J Immunol 1972;108:1624-1630.

32. Dhariwala FA, Narang H, Krishna M. Differential response of three cell types to dual stress of nitric oxide and radiation. Cell Biol Toxicol 2012;28:161-173.

33. Khan SZ, Hand N, Zeichner SL. Apoptosis-induced activation of HIV-1 in latently infected cell lines. Retrovirology 2015;12:42.

34. Allers K, Hutter G, Hofmann J, et al.: Evidence for the cure of HIV infection by CCR5Delta32/Delta32 stem cell transplantation. Blood 2011;117:2791-2799.

35. Henrich TJ, Hu Z, Li JZ, et al.: Long-term reduction in peripheral blood HIV type 1 reservoirs following reducedintensity conditioning allogeneic stem cell transplantation. J Infect Dis 2013;207:1694-1702.

36. Yukl SA, Boritz E, Busch M, et al.: Challenges in detecting HIV persistence during potentially curative interventions: A study of the Berlin patient. PLoS Pathog 2013;9:e1003347.

37. Dadachova E, Patel MC, Toussi S, et al.: Targeted killing of virally infected cells by radiolabeled antibodies to viral proteins. PLoS Med 2006;3:e427.

38. Dadachova E, Kitchen SG, Bristol G, et al: Pre-clinical evaluation of a 213Bi-labeled 2556 antibody to HIV-1 gp41 glycoprotein in HIV-1 mouse models as a reagent for HIV eradication. PLoS One 2012;7:e31866.

39. Li CJ, Wang C, Friedman DJ, Pardee AB: Reciprocal modulations between p53 and Tat of human immunodeficiency virus type 1. Proc Natl Acad Sci U S A 1995;92:5461-5464.

40. Gabizon R, Mor M, Rosenberg MM, et al.: Using peptides to study the interaction between the p53 tetramerization domain and HIV-1 Tat. Biopolymers 2008;90:105-116.

41. Van Duyne R, Easley R, Wu W, et al.: Lysine methylation of HIV-1 Tat regulates transcriptional activity of the viral LTR. Retrovirology 2008;5:40.

42. Sivakumaran H, van der Horst A, Fulcher AJ, et al:: Arginine methylation increases the stability of human immunodeficiency virus type 1 Tat. J Virol 2009;83:11694-11703.

43. Sakane N, Kwon HS, Pagans S, et al.: Activation of HIV transcription by the viral Tat protein requires a demethylation step mediated by lysine-specific demethylase 1 (LSD1/KDM1). PLoS Pathog 2011;7:e1002184.

44. Sampey GC, Saifuddin M, Schwab A, et al.: Exosomes from HIV-1 infected cells stimulate production of pro-inflammatory cytokines through TAR RNA. J Biol Chem 2016;291:12511266.

45. Roshal M, Kim B, Zhu Y, Nghiem P, Planelles V: Activation of the ATR-mediated DNA damage response by the HIV-1 viral protein R. J Biol Chem 2003;278:2587925886.

46. Zimmerman ES, Sherman MP, Blackett JL, et al:: Human immunodeficiency virus type $1 \mathrm{Vpr}$ induces DNA replication stress in vitro and in vivo. J Virol 2006;80:10407-10418.

47. Vassena L, Giuliani E, Matusali G, Cohen EA, Doria M. The human immunodeficiency virus type $1 \mathrm{Vpr}$ protein upregulates PVR via activation of the ATR-mediated DNA damage response pathway. J Gen Virol 2013;94:2664-2669.

48. Klase Z, Winograd R, Davis J, et al.: HIV-1 TAR miRNA protects against apoptosis by altering cellular gene expression. Retrovirology 2009;6:18.
49. Longo F, Marchetti MA, Castagnoli L, Battaglia PA, Gigliani F: A novel approach to protein-protein interaction: complex formation between the p53 tumor suppressor and the HIV Tat proteins. Biochem Biophys Res Commun 1995;206:326-334.

50. Zhou BY, He JJ: Proliferation inhibition of astrocytes, neurons, and non-glial cells by intracellularly expressed human immunodeficiency virus type 1 (HIV-1) Tat protein. Neurosci Lett 2004;359:155-158.

51. Chen BP, Chan DW, Kobayashi J, et al.: Cell cycle dependence of DNA-dependent protein kinase phosphorylation in response to DNA double strand breaks. J Biol Chem 2005;280:14709-14715.

52. Nagasawa M, Watanabe F, Suwa A, Yamamoto K, Tsukada $\mathrm{K}$, Teraoka H: Nuclear translocation of the catalytic component of DNA-dependent protein kinase upon growth stimulation in normal human $\mathrm{T}$ lymphocytes. Cell Struct Funct 1997;22:585-594.

53. Brenchley JM, Schacker TW, Ruff LE, et al.: CD4+ T cell depletion during all stages of HIV disease occurs predominantly in the gastrointestinal tract. J Exp Med 2004; 200:749-759.

54. Shao L, Goronzy JJ, Weyand CM: DNA-dependent protein kinase catalytic subunit mediates T-cell loss in rheumatoid arthritis. EMBO Mol Med 2010;2:415-427.

55. Hill R, Lee PW: The DNA-dependent protein kinase (DNA$\mathrm{PK})$ : More than just a case of making ends meet? Cell Cycle 2010;9:3460-3469.

56. Callen E, Jankovic M, Wong $\mathrm{N}$, et al.: Essential role for DNA-PKcs in DNA double-strand break repair and apoptosis in ATM-deficient lymphocytes. Mol Cell 2009;34:285-297.

57. Cooper A, Garcia M, Petrovas C, Yamamoto T, Koup RA, Nabel GJ: HIV-1 causes CD4 cell death through DNAdependent protein kinase during viral integration. Nature 2013;498:376-379.

58. Durkin SS, Guo X, Fryrear KA, et al.: HTLV-1 Tax oncoprotein subverts the cellular DNA damage response via binding to DNA-dependent protein kinase. J Biol Chem 2008;283:36311-36320.

59. Chandhasin C, Ducu RI, Berkovich E, Kastan MB, Marriott SJ: Human T-cell leukemia virus type 1 tax attenuates the ATM-mediated cellular DNA damage response. J Virol 2008;82:6952-6961.

60. Marriott SJ, Semmes OJ: Impact of HTLV-I Tax on cell cycle progression and the cellular DNA damage repair response. Oncogene 2005;24:5986-5995.

61. Park HU, Jeong JH, Chung JH, Brady JN: Human T-cell leukemia virus type 1 Tax interacts with Chk1 and attenuates DNA-damage induced G2 arrest mediated by Chk1. Oncogene 2004;23:4966-4974.

62. Majone F, Jeang KT: Unstabilized DNA breaks in HTLV-1 Tax expressing cells correlate with functional targeting of Ku80, not PKcs, XRCC4, or H2AX. Cell Biosci 2012;2:15.

Address correspondence to: Fatah Kashanchi, PhD Laboratory of Molecular Virology School of Systems Biology George Mason University

Discovery Hall, Room 182 10900 University Blvd. MS $1 \mathrm{H} 8$ Manassas, VA 20110

E-mail: fkashanc@gmu.edu 